\title{
PMN-PT based Smart Sensing System for Viscosity and Density Measurement
}

\author{
Chen Zhang ${ }^{1}$, Suresh Kaluvan ${ }^{2}$, Haifeng Zhang (Corresponding Author) ${ }^{2}$, Guoan Wang ${ }^{3}$
}

${ }^{1}$ Department of Mechanical and Energy Engineering, University of North Texas, Denton, Texas, 76203, USA

${ }^{2}$ Department of Engineering Technology, University of North Texas, Denton, Texas, 76203, USA

Corresponding Author: Dr. Haifeng Zhang, Email: haifeng.zhang@unt.edu, Tel: 940369-8266, Fax: 940-565-2666

Department of Electrical Engineering, University of South Carolina, Columbus, SC, 29208

\begin{abstract}
This paper presents a smart sensing system for viscosity and density measurement of viscous fluids. The proposed system is based on the vibrational properties of a cantilever probe bonded with newly developed piezoelectric materials-Lead Magnesium Niobate/Lead Titanate (PMN-PT) transducer, which has a piezoelectric charge constant (d33) more than 3 times larger than that of traditional piezoelectric material Lead zirconate titanate (PZT). The proposed system utilizes a PMN-PT single crystal for actuation and laser vibrometer for vibration detection. Using the PMN-PT transducer, the measured viscosity and density of fluids were extracted by analyzing the vibrational properties of the smart probe. Finite element analysis was conducted with COMSOL Multiphysics for theoretical calculation and lab tests were carried out to verify and evaluate the simulation results. This smart sensing system can be applied to in-field, in situ and real-time monitoring of viscous fluids, such as blood, engine oil and early-age concrete.
\end{abstract}

Keyword: PMN-PT, viscosity, density, FEA, COMSOL, frequency response.

\section{Introduction}

In fluid mechanics and engineering, viscosity plays an extremely important role in understanding and determining a fluid's characteristics and performance, especially in viscous fluid engineering such as petroleum pipelines, the food industry and automotive industry. Therefore, the topic of real-time monitoring of viscosity variation is considered by researchers to be one of the key factors to improve feedback control strategy and entire hydraulic performance. Researchers have made efforts to develop effective systems/approaches of real-time viscosity monitoring. Kong et al. [1] proposed an active sensing system that employs a pair of sandwich structure PZT patches as an actuator and sensor, and monitoring was implemented by analyzing the change of transferred electrical signals. Franco et al. [2] conducted experiments to aluminum and applied shear-wave coefficient to monitor the very early concrete viscosity change along with a novel signal processing technique which is mainly focused on the calculation of reflection coefficient magnitude in frequency band. Lu et al. [3] developed out a new method that involves the application of embedded piezoelectric sensors so that monitoring can be determined by 
analyzing the velocity curves. Wilkie-Chancelie et al. [4] designed an innovative sensor that is contacted to the viscous material and employs a reflected Lamb wave at the interface as the index to indicate the viscosity change of the material.

Piezoelectric-based sensors are considered adaptive for viscosity monitoring due to its quick-response, high-accuracy, and low-maintenance needs. Wilkie-Chancellier et al. [4] used a piezoelectric plate as the sensor and applied guided wave (Lamb wave) to monitor the mechanical impedance and then obtain the viscosity of the tested material. This method was found to be valid only when the tested material is a weak fluid, that is, it should have Newtonian properties. However, most viscous fluids are not that "weak" and their properties change with working condition and time, so this method is not adaptive for in situ monitoring. In addition, ultrasound propagation in viscous fluids is too complicated to be analyzed. Ultrasound will diffuse, dissipate, and scatter at boundaries between different phases and generate lots of noises.

In the authors' previous work, a series of experiments were conducted to verify the effectiveness of the prototype of a PZN-PT-based viscosity sensor for viscosity monitoring at elevated temperatures. Results show that the viscosity is an expression of the vibrational velocity of the sensing probe [5]. In this paper, a PMN-PT based viscosity sensor is designed and experimentally tested at room temperature and advanced finite element analysis is carried out for theoretical calculation of both viscosity and density measurement.

\section{PMN-PT Single Crystal based Smart Probe}

\subsection{PMN-PT single crystal}

The single crystals of lead magnesium niobate-lead titanate solid solution, $\left[\mathrm{Pb}\left(\mathrm{Mg}_{1 / 3} \mathrm{Nb}_{2 / 3}\right) \mathrm{O}_{3}\right]_{(1-\mathrm{x})}-[\mathrm{PbTiO} 3]_{\mathrm{x}}(\mathrm{PMN}-\mathrm{PT})$, are a new generation of piezoelectric materials. The new material was formulated to exhibit very large electromechanical coupling coefficients, high piezoelectric coefficients, high dielectric constants, and low dielectric losses that results in improving bandwidth, sensitivity, and source level in applications [6]. Comparing to the traditional PZT material (i.e. PZT-4), PMN-PT has a higher electromechanical coefficient as well as a better $\mathrm{Q}$ factor which provides a narrow bandwidth frequencies and avoid noises [7].

Single crystal PMN-PT also has exceptional properties and is poised to revolutionize applications from medical ultrasound to adaptive optics and energy harvesting. The crystal exhibits five times of the strain energy density and significantly higher electromechanical coupling than conventional piezo-ceramics [8].

Fig. 1 shows the picture of PMN-PT single crystal plate resonator and Table 1 lists the main properties [9]. In comparison to other piezoelectric materials, PMN-PT has a larger coupling coefficient around 0.87 0.94 which will provide better piezoelectric effect than other piezoelectric materials. 


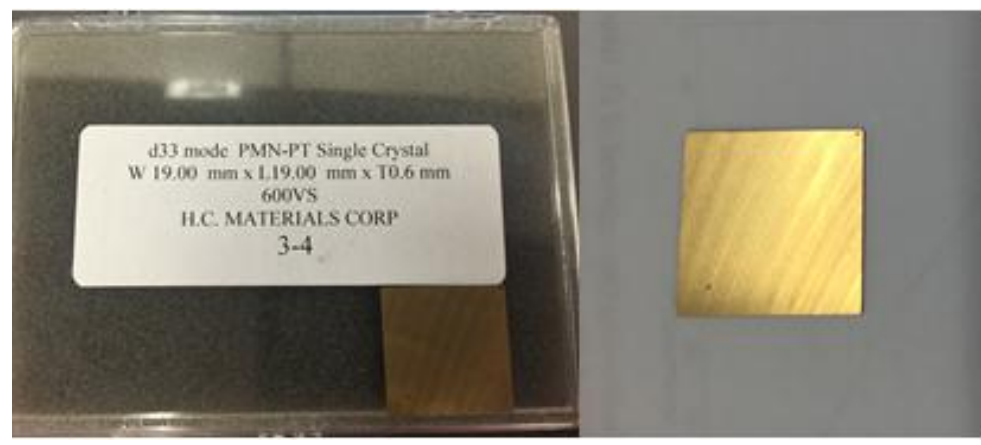

Fig. 1 PMN-PT single crystal plate resonator

Table 1 Major properties of PMN-PT single crystals

\begin{tabular}{ccc}
\hline PROPERTIES & $\begin{array}{c}\text { PMN-PT-A }(\mathrm{PT}=0.27 \sim 0.30) \\
\text { Fot Large Signal }\end{array}$ & $\begin{array}{c}\text { PMN-PT-B (PT }=0.30 \sim 0.33) \\
\text { For Small Signal }\end{array}$ \\
\hline $\mathrm{K}_{33}{ }^{\mathrm{T}} @ 20 \mathrm{C}$ & $4500 \sim 5500$ & $5500 \sim 6500$ \\
$\mathrm{~d}_{33}{ }^{\mathrm{T}}(\mathrm{pC} / \mathrm{N})$ & $1400 \sim 2000$ & $2000 \sim 3500$ \\
Coupling Coefficient $k_{33}$ & $0.87 \sim 0.90$ & $0.90 \sim 0.94$ \\
$\mathrm{Y}_{33}{ }^{\mathrm{E}}(\mathrm{GPa})$ & $20 \sim 25$ & $16 \sim 20$ \\
$\mathrm{E}_{\mathrm{c}}(\mathrm{V} / \mathrm{mm})$ & 300 & 250 \\
$\tan \boldsymbol{(}\left(1 \mathrm{kHz}, 20{ }^{\circ} \mathrm{C}\right)$ & $<0.005$ & $<0.008$ \\
Depolarization & $>90$ & $>75$ \\
Temperature $\left({ }^{\circ} \mathrm{C}\right)$ & & 0.0025 \\
$\begin{array}{c}\text { Thermal Conductivity } \\
(\mathrm{W} / \mathrm{cm} . \mathrm{K})\end{array}$ & 0.0026 & $>10$ \\
$\begin{array}{c}\text { Thermal Expansion } \\
\text { Coefficient } 10^{-6} /{ }^{\circ} \mathrm{C}\left(20 \sim 70{ }^{\circ} \mathrm{C}\right)\end{array}$ & 9.5 & \\
\hline \hline
\end{tabular}

2.2 PMN-PT Based smart viscosity probe

The dimension and the schematic representation of the smart probe is given in Fig. 2. A PMN-PT single crystal patch resonator was bonded on the aluminum cantilever with permanent nonconductive glue. Thickness mode $\left(\mathrm{d}_{33}\right)$ of the PMN-PT piezo material is applied as an actuation to excite an effective transverse mode when the free end of the cantilever immersed into the viscous fluid.

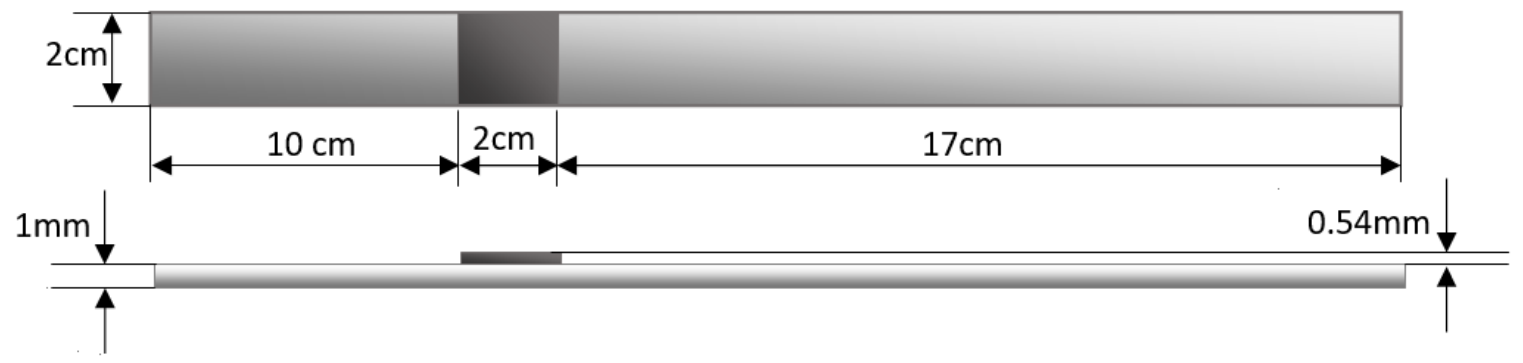

Fig. 2 Schematic representation of the smart probe 


\section{Finite Element Modeling}

When cantilever immersed into the viscous fluid, its resonant frequency and vibrational velocity are changed as a part of damping effects. Generally, the resonant frequency are affected by Eq.1 [10],

$$
\omega_{\text {fluid }}=\frac{\omega_{\text {air }}}{\sqrt{1+\frac{\pi b}{4 h} \frac{\rho_{\text {fluid }}}{\rho_{\text {beam }}} \tau}}
$$

Where $b$ is the width of the cantilever, $h$ is the length of the cantilever and $\tau$ is the correction factor while the peak-to-peak velocity is an expression of the viscosity, which is in terms of loss factor that can be extracted by logarithmic decrement method. However, different from the typical case, by which the entire cantilever is immersed in the viscous fluid, the proposed approach in this paper in more complex, so a FEM is carried out to provide a numerical simulation.

Fig. 3 shows the 3D finite element modeling with COMSOL Multiphysics. The problem is set up as a coupled acoustic-structure-electrostatics eigenvalue analysis. In this study, there are three physics in this study, Solid Mechanics, Electrostatics and Acoustic-Structure. The cantilever is involved in the Solid Mechanics physics. A fixed constraint is added to one end of the cantilever while the other end is immersed in the fluid and free to vibrate. The PMN-PT single crystal is subject to a Multiphysicspiezoelectric effect, which includes both Solid Mechanics and Electrostatics. Excitation voltage is applied to the top surface of the PMN-PT single crystal while the bottom surface is set as ground. Hard sound boundaries is setup as default in COMSOL Multiphysics to the viscous fluid. Frequency domain analysis is also added to the study. FEA analysis is conducted with 3 different types of engine oil and Table. 2 gives the properties of them. Figure.4 shows the results of the frequency domain analysis in COMSOL Multiphysics.

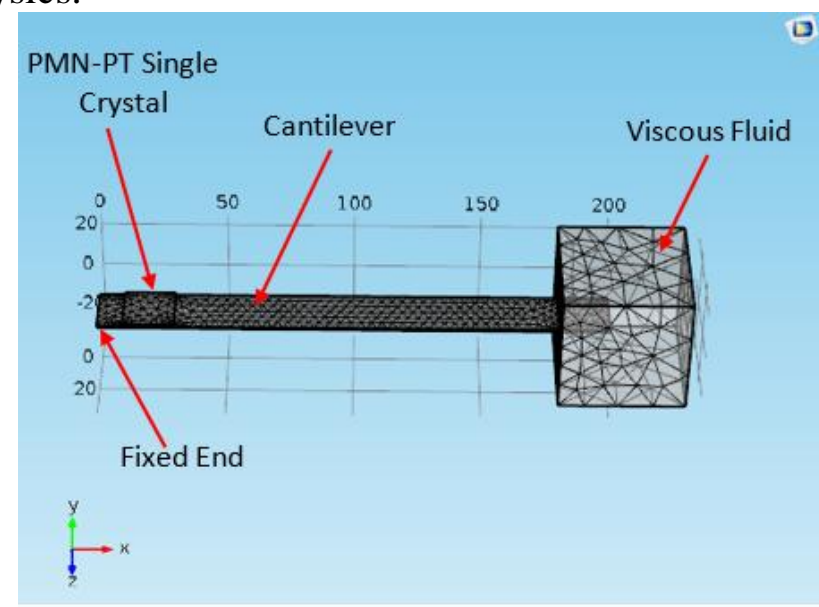

Fig.3 3D finite element modeling in COMSOL Multiphysics.

Table 2 Properties of tested engine oil.

\begin{tabular}{|c|c|c|c|c|}
\hline Type & $\begin{array}{c}\text { Temperature } \\
{\left[{ }^{\circ} \mathrm{C}\right]}\end{array}$ & $\begin{array}{c}\text { Dyn. Viscosity } \\
{[\mathrm{mPa} . \mathrm{s}]}\end{array}$ & $\begin{array}{c}\text { Density } \\
{\left[\mathrm{g} / \mathrm{cm}^{\wedge} 3\right]}\end{array}$ & Loss Factor \\
\hline SAE 15W-40 & 20 & 287.23 & 0.8787 & 0.3 \\
\hline SAE 10W-60 & 20 & 381.08 & 0.8504 & 0.45 \\
\hline SAE 30 & 20 & 239.39 & 0.8815 & 0.23 \\
\hline
\end{tabular}




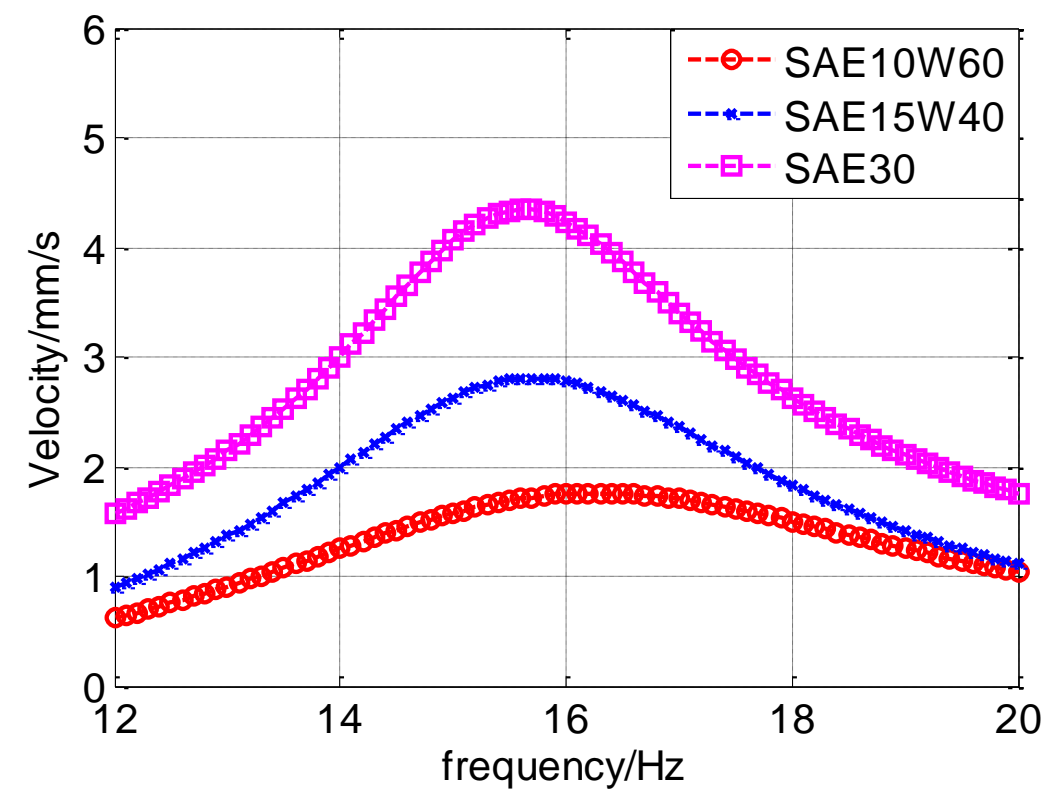

Fig.4 FEA simulation result of the frequency responses of the smart probe

\section{Experimental Analysis}

\subsection{Experimental setup}

The experiment is conducted with LabVIEW version 2015. The cantilever is fixed at one end while the other end is free to vibrate inside the viscous fluid. A sinusoidal wave is applied to generate a mechanical vibration (Agilent 33220A, Amplitude: 5volts, Frequency: sweeping from $10 \mathrm{~Hz}$ to $22 \mathrm{~Hz}$ with a resolution of $0.1 \mathrm{~Hz}$ ). The peak-to-peak velocity is measured at the point that has a distance of $d(=1 \mathrm{~cm})$ from the free end by a laser vibrometer (Polytec OFV-505). The frequency response is acquired by a digital oscilloscope (Tektronix TDS 3054C) and then sent to the computer for further processing [5]. The schematic diagram of the experiment is given in Fig. 5 while Fig. 6 shows the experimental setup.

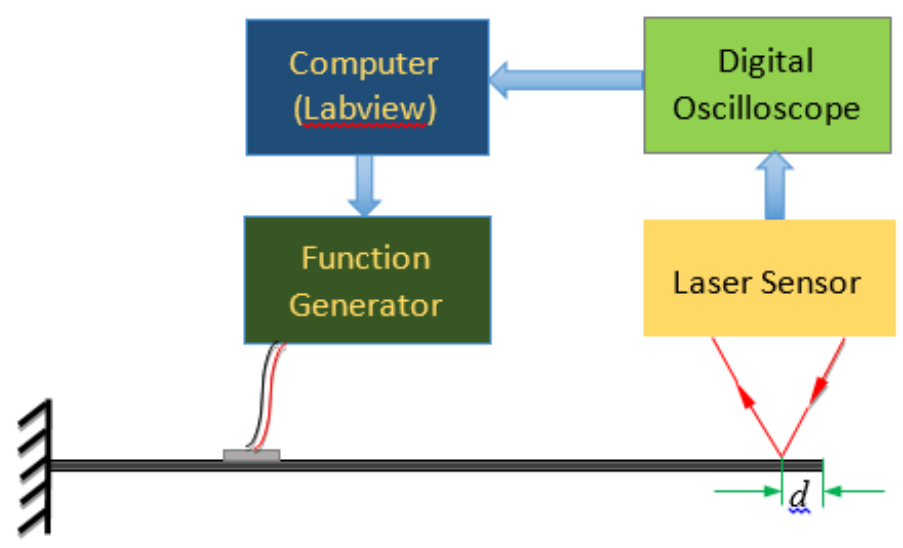

Fig. 5 Schematic diagram of the experiment 


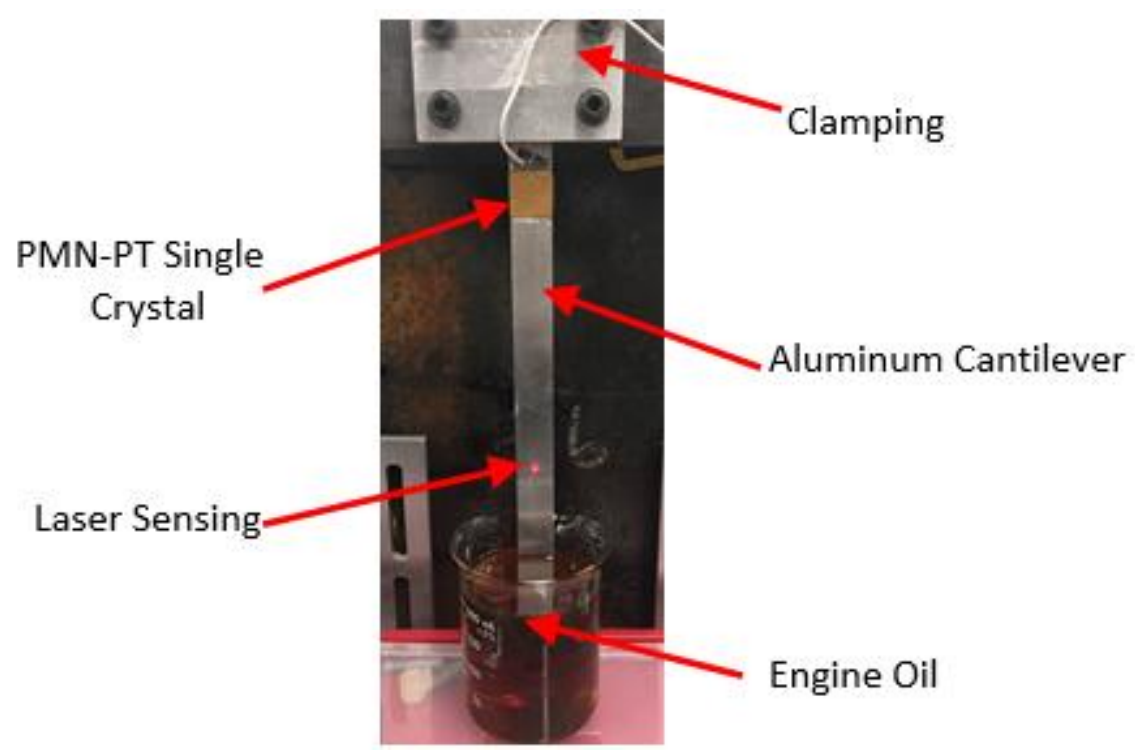

Fig. 6 Photograph of PMN-PT based viscometer

\subsection{Results and discussion}

Lab tests were carried out to evaluate the frequency response of the proposed sensing system and to verify the FEA results. The results obtained in this analysis are also associated with the results given in paper $[5,11]$. The detailed frequency responses are shown in Fig. 7.

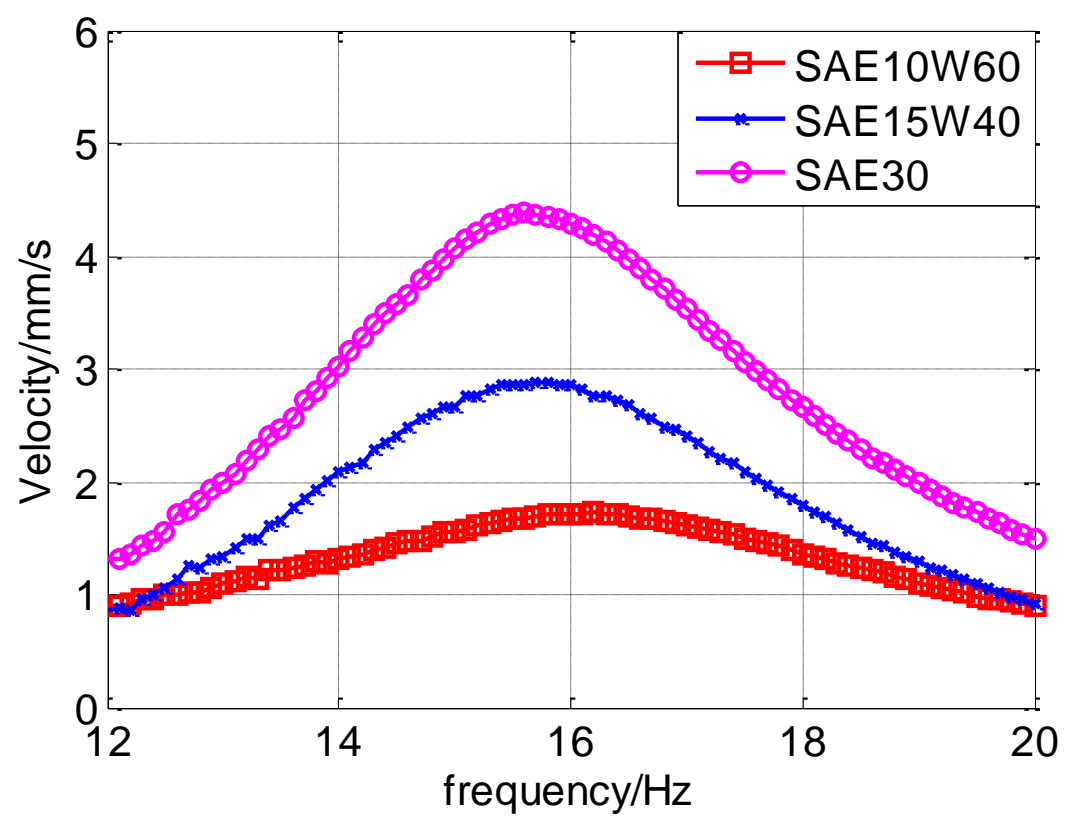

Fig.7 Experiment results of the frequency responses of the smart probe

As shown in Fig. 4 and Fig. 7, when cantilever vibrating in the viscous fluid, its resonant frequency will be affected by the effect of adding mass (density ration in Eq.1). Meanwhile, its peak-to-peak velocity is actually influenced by the damping effect. Considering the internal damping is keeping constant during the experiment (the same cantilever), the peak-to-peak velocities of the cantilever is therefore determined by the viscous damping effect (external damping), which shows a significant decreasing 
tendency when increasing the viscosity of the fluid. The results are also associated with the given paper $[5,11]$. Figure 8 shows the comparison between FEA and experiment results. It is clear that for each type of engine oil, the resonant frequency as well as peakto-peak velocity is matched between FEA and experiment results.

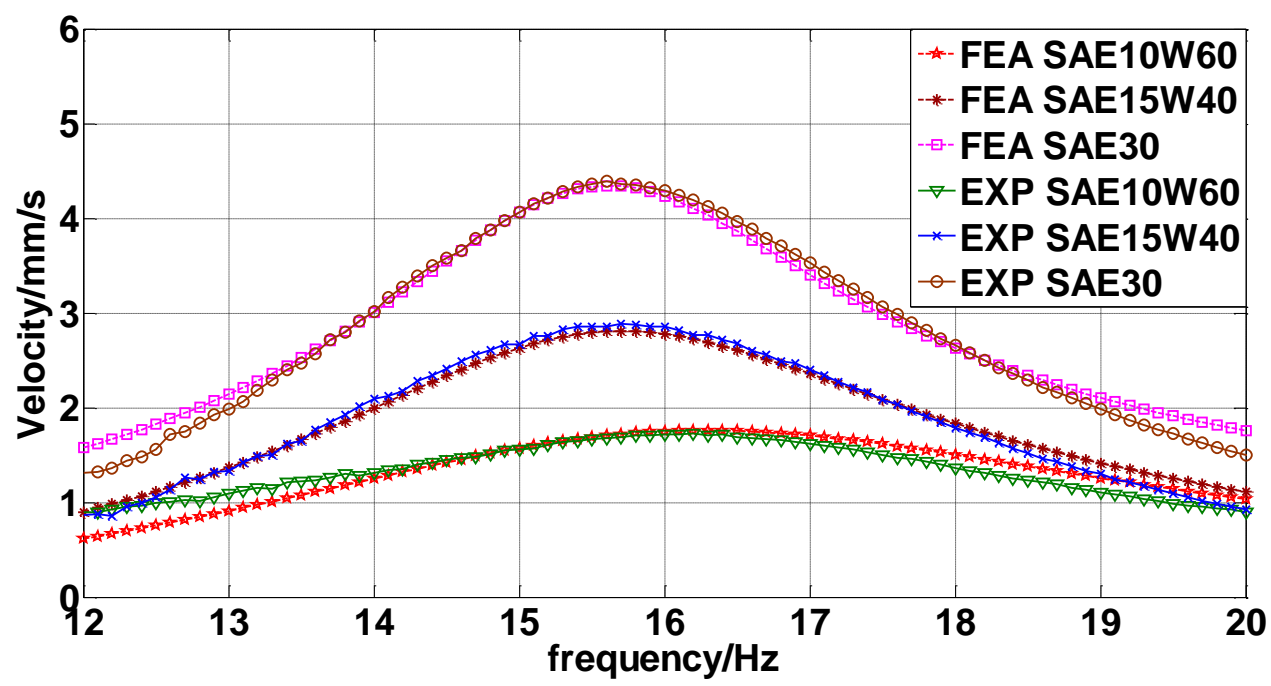

Fig. 8 Comparison of between FEA and experiment results

Specifically, Fig. 9 shows the resonant frequency comparison between FEA and experiment results. The resonant frequency is decreasing with the increase of the density. While Fig. 10 shows the peak-to-peak velocity comparison between FEA and experiment results, which suggests that when increasing the viscosity, the peak-to-peak velocity will be reduced. The error between experiment and simulation is due to the slight difference between the reality and ideal case, like, environment temperature, immersing depth of the cantilever, which is minor and could be ignored.

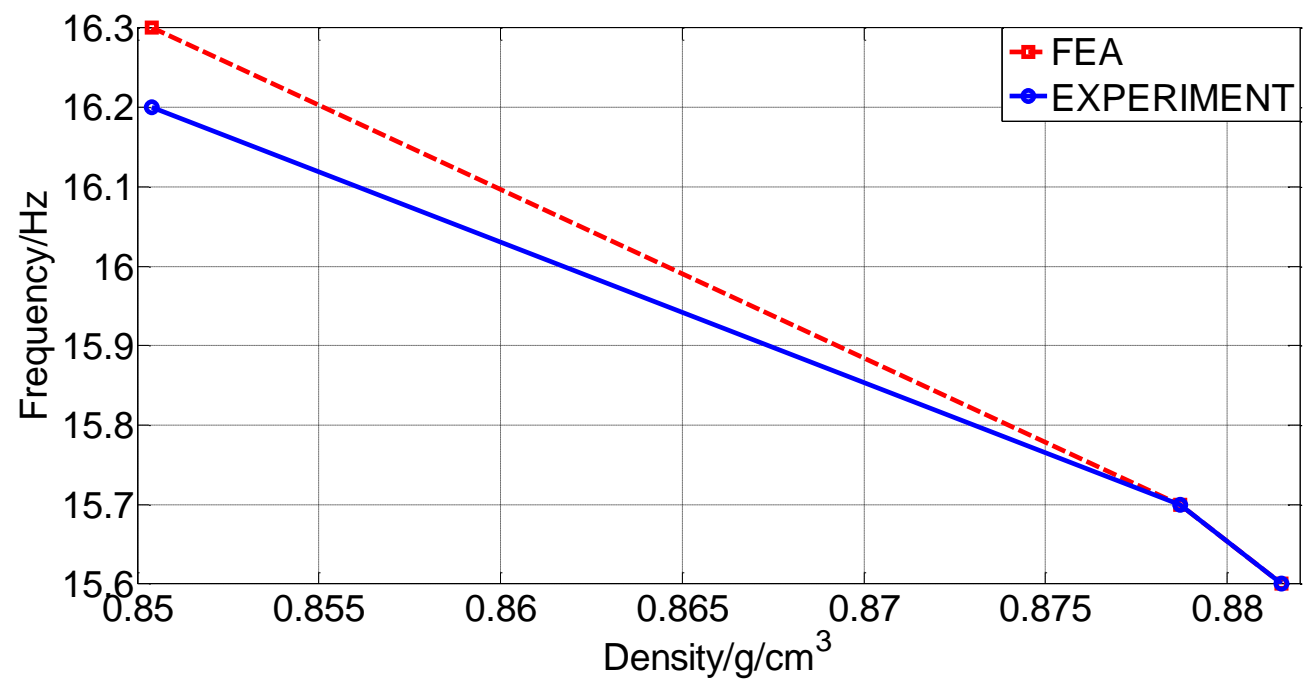

Fig. 9 Comparison in Resonant Frequency vs Density 


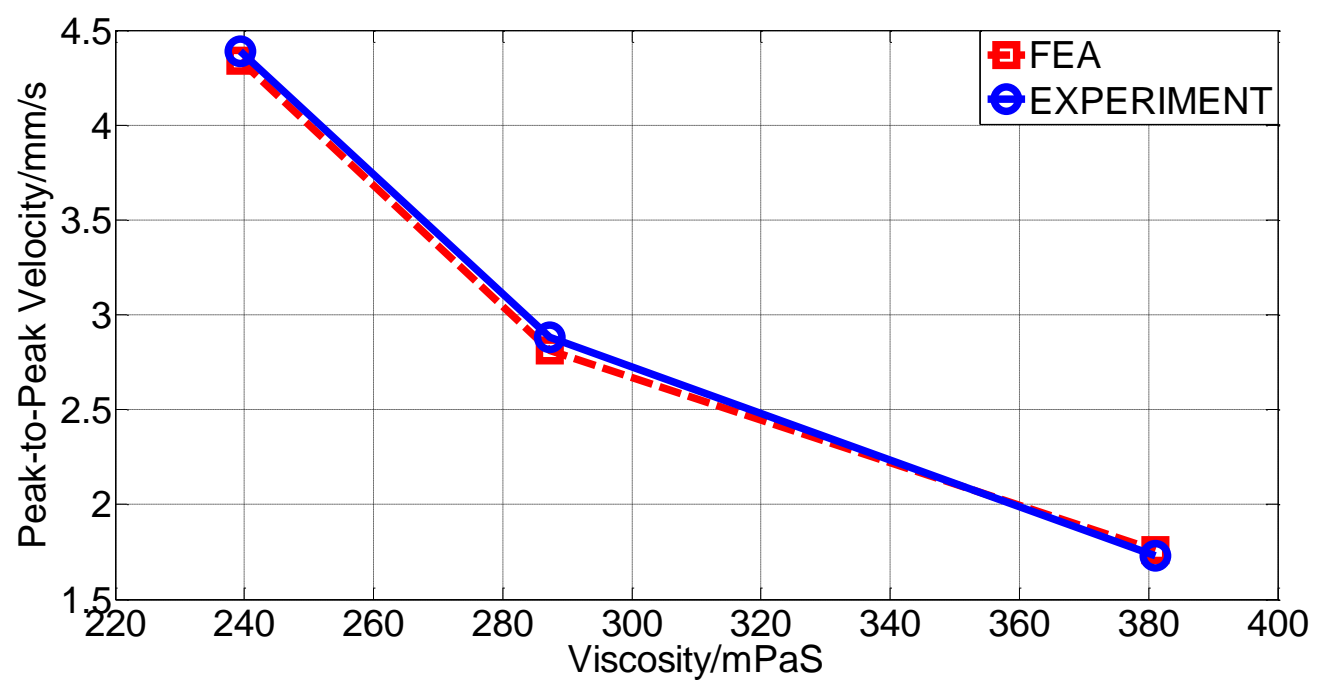

Fig. 10 Comparison in Peak-to-Peak Velocity vs Viscosity

\section{Conclusion}

Smart sensing system with new generation piezoelectric material, PMN-PT, has been an attractive topic for its high electromechanical coupling coefficient and better quality factor. In this paper a piezoelectric smart sensing system is fabricated with a PMN-PT single crystal. FEA analysis and lab test were carried out to verify the proposed sensing system. Different from other existing viscometer, PMN-PT based piezoelectric sensing system can implement the density and viscosity measurement of the viscous fluid simultaneously by investigating the frequency response and the peak-to-peak velocity of the cantilever. Results shows that the proposed PMN-PT based smart sensing system will have a frequency shift with density change, while a peak-to-peak velocity change is observed due to viscosity change. The proposed smart sensing system is sensitive and easy to implement and can be applied real-time viscosity and density monitoring.

\section{Acknowledgement}

This research is sponsored by National Science Foundation under award number: 1508711.

\section{References:}

[1] Qingzhao Kong, Shuang Hou, Qing Ji, Y L Mo and Gangbing Song (2013). "Very early age concrete hydration characterization monitoring using piezoceramic based smart aggregates". Smart Material and Structure, 22 (2013): 085025 (7pp).

[2] Ediguer E. Franco, Julio C. Adamowski and Flávio Buiochi (2010). "Ultrasonic Viscosity Measurement Using the Shear-Wave Reflection Coefficient with a Novel Signal Processing Technique". IEEE Transactions on Ultrasonics, Ferroelectrics, and Frequency Control, 55 (2010): 1133-1139.

[3] Youyuan Lu, Hongyan Ma and Zongjin Li (2014). "Ultrasonic monitoring of the early-age hydration of mineral admixtures incorporated concrete using cement 
based piezoelectric composite sensors". Journal of Intelligent Material Systems and Structures, DOI: 10.1177/1045389X14525488

[4] Nicolas Wilkie-Chancellier, Loïc Martinez, Stéphane Serfaty, Member IEEE, and Pascal Griesmar [2009]. "Lamb Wave Sensor for Viscous Fluids Characterization". IEEE Sensors Journal, 9 (2009): 1142-1147.

[5] Chen Zhang, Haifeng Zhang and Suresh Kaluvan (2016). "PZN-PT based smart probe for high temperature fluid viscosity measurements". Measurement, 94 (2016): 753-758.

[6] http://www.sinocera.net/en/crystal_pmn.asp

[7] Shujun Zhang, Fei Li, Nevin P. Sherlock, Jun Luo, Hyeong Jae Lee, Ru Xia, Richard J. Meyer, Jr, Wesley Hackenberger and Thomas R. Shrout (2011). "Recent Developments on High Curie temperature PIN-PMN-PT Ferroelectric Crystals". Journal of Crystal Growth, 318(1): 846-850.

[8] http://www.trstechnologies.com/Materials/High-Performance-PMN-PT Piezoelectric-Single-Crystal

[9] http://www.hcmat.com/Pmn_Properties.html

[10] https://www.comsol.com/blogs/natural-frequencies-immersed-beams/

[11] Matthew D. Brouwer, Lokesh A. Gupta, Farshid Sadeghi, Dimitrios Peroulis and Douglas Adams (2012). "High temperature dynamic viscosity sensor for engine oil applications". Sensors and Actuators A: Physical, 173(2012): 102-107. 